\title{
Fatigue and Depression from Early Postpartum to 1 Month among Postpartum Women with Mental Disorders
}

\author{
Nozomi Detsuka1, Ai Kawashima ${ }^{2}$, Rika Yano2* \\ ${ }^{1}$ Kin-ikyo Sapporo Hospital, Sapporo, Japan \\ ${ }^{2}$ Faculty of Health Sciences, Hokkaido University, Sapporo, Japan \\ Email: ${ }^{\star}$-yano@med.hokudai.ac.jp
}

How to cite this paper: Detsuka, N., Kawashima, A. and Yano, R. (2017) Fatigue and Depression from Early Postpartum to 1 Month among Postpartum Women with Mental Disorders. Open Journal of Nursing, 7, 1430-1438.

https://doi.org/10.4236/ojn.2017.712101

Received: November 29, 2017

Accepted: December 23, 2017

Published: December 26, 2017

Copyright (c) 2017 by authors and Scientific Research Publishing Inc. This work is licensed under the Creative Commons Attribution International License (CC BY 4.0).

http://creativecommons.org/licenses/by/4.0/

\begin{abstract}
Objective: This study aims to ascertain the prevalence of fatigue and depression from early postpartum to 1 month after delivery among postpartum women with mental disorders. Methods: The participants of this study were postpartum women who had delivered a child after a full-term pregnancy and were suffering from a mental disorder. We administered the Postpartum Fatigue Scale (PFS) on days 1,3 , and 4 (to multiparas), and 1, 3, and 5 (to primiparas), and 1 month after delivery to all participants. The Edinburgh Postnatal Depression Scale (EPDS), Japanese version, was also administered at the same time intervals as PFS except on day 3 after delivery. Results: A total of 7 primiparas and 9 multiparas participated in this study. The average age was $29.0 \pm 7.0$ years in primiparas and $32.0 \pm 4.0$ years in multiparas. The PFS scores were higher among the primiparas and peaked at day 1 , and more than half the primiparas scored more than 9 points in EPDS. In addition, the EPDS score at 1 month had a strong correlation with the PFS subscale "mental stress situation" ( $\mathrm{r}=0.818 / p=0.047)$. In multiparas, the percentage of women who scored more than 9 points decreased. However, their EPDS scores at 1 month were related to the total scores of the PFS, "physical stress situation", "mental stress situation", and "sleep deprivation situation". Especially, the score of "sleep deprivation situation" was higher than those during hospitalization. Conclusion: There was more fatigue 1 month after the discharge among postpartum women with mental disorders. The percentage of women whose EPDS scores were more than 9 points was high in both primiparas and multiparas. This study suggests assisting primiparas and multiparas by controlling the former's disorder during childcare, and using family support for the latter, so that they get time to rest.
\end{abstract}




\section{Keywords}

Fatigue, Depression, Mental Disorder, Postpartum Women

\section{Introduction}

According to the report of the Ministry of Health, Labor and Welfare (2016) [1], it has been found that in recent years the percentage of pregnant women with mental disorders has remained constant at around 2.5\%. Some mental disorders associated with pregnant women are depression and schizophrenia. Pregnant women with mental disorders are likely to gain more weight during pregnancy, depending on the type of drug they are taking, and have more difficulty in controlling drinking and smoking habits as compared to pregnant women who do not have mental disorders [2]. It is common for postpartum depression to cause social dysfunction in mothers that affects their children and families [3]. Therefore, in the case of women with mental disorders there is a high risk during pregnancy and childbirth, and to the newborn babies. History of mental disorder is also a risk factor for the development of postpartum depression as a mental problem [4]. As the incidence of mental disorders has been increasing, especially mood disorders have seen a rising trend recently [5], it is estimated that the number of pregnant women with mental disorders will inevitably increase. According to Tsuchiya et al.'s report [6], the likelihood for postpartum depression in mothers with mental disorders is related to a history of smoking during pregnancy, hypertensive disorders of pregnancy, unplanned pregnancy, not taking rest, and kind of delivery. In addition to that, early postpartum is a period when fatigue is likely to occur due to burden of childbirth and child rearing, and particularly women with mental disorders suffer from fatigue more severely than other pregnant women and this seems to be the cause of their depressed state. Bozoky \& Corwin [7] discuss the relationship between fatigue and depression and postpartum, but early postpartum women with mental disorders have not yet been studied in the relation to fatigue and depression. Therefore, the purpose of this study was to examine fatigue and depression from early postpartum to 1 month in women with mental disorders. We also propose that palliative care for fatigue can be suggested for prevention of postpartum depression in women with mental disorders.

\section{Methods}

\subsection{Participants}

The participants were postpartum women with mental disorders who had given birth to a singleton baby, with no critical disease at birth, at a hospital's obstetrics and gynecology department from May 2015 to April 2016. We selected women with mental disorders according to the International Classification of Diseases 10th Edition (ICD-10). The women who gave their consent by signing a 
document were selected for the study.

\subsection{Survey Method}

From day 0 to 1 after birth, the research or hospital staff explained the purpose of the study verbally as well as by handing out reading material to the women who met the target criteria.

\subsection{Data Collection}

1) Postpartum fatigue: The Postpartum Fatigue Scale (PFS) was used for analysis [8]. It is a four-point scale consisting of 36 items and is divided into four subscales, namely, "physiological stress situation", "mental stress situation", "sleep deprivation situation", and "feeling of difficulty in childrearing". Each of these subscales includes nine items, and the score range is 9 to 36 points, and the total PFS score can range from 36 to 144 points. The higher the score, the stronger is the postpartum fatigue that she is said to experience. The PFS was administered on days 1,3 , and 4 (for multiparas), days 1,3 , and 5 (for primiparas), and 1 month after birth to all participants, along with a 1-month health examination.

2) Depression: The Edinburgh Postnatal Depression Scale (EPDS), Japanese version [9], was used to measure depression. EPDS is a four-point scale consisting of 10 items. In Japan, high validity is indicated when the division point of the EPDS score is set to 8/9 points. The EPDS was administered on days 1 and 4 (to multiparas) and days 1 , and 5 (to primiparas), and 1 month after birth to all participants, along with 1-month health examination.

\subsection{Data Analysis}

Descriptive statistics of demographic data, PFS points, and EPDS scores were sought for primiparas and multiparas. For primipara's EPDS, one of the participants was missing, so she was excluded from the EPDS analysis. After confirming normality, the significance of the correlation between the PFS and the EPDS scores was confirmed by Pearson's correlation coefficient or Spearman's rank correlation coefficient. The statistical software SPSS version 24 was used for analysis, and the significance probability was set to $5 \%$.

\subsection{Ethical Considerations}

We received the participants' consent verbally as well on a signed document. We explained the purpose and method of research and optional participation (i.e., being able to withdraw in between the study) to them, ensuring confidentiality of their personal information. This study was conducted with the approval of the Ethics Committee of the hospital.

\section{Results}

\subsection{Characteristics of Participants}

Table 1 shows the characteristics of the participants. The mean age of the 
Table 1. Participant characteristics.

\begin{tabular}{|c|c|c|c|}
\hline & & $\begin{array}{l}\text { Primiparas } \\
\quad(\mathrm{n}=7)\end{array}$ & $\begin{array}{l}\text { Multiparas } \\
\quad(\mathrm{n}=9)\end{array}$ \\
\hline Age, $y$, Mean \pm SD & & $29.0 \pm 7.0$ & $32.0 \pm 4.0$ \\
\hline \multirow{2}{*}{ Marital status } & Married & 4 & 4 \\
\hline & Single & 3 & 5 \\
\hline \multirow{2}{*}{ Employment } & Employed & 1 & 3 \\
\hline & Not employed & 6 & 6 \\
\hline \multirow{2}{*}{ Education } & Junior or high school & 5 & 8 \\
\hline & Vocational school or college & 2 & 1 \\
\hline Birth weight $(\mathrm{g})$, Mean \pm SD & & $2949.7 \pm 290.6$ & $2925.8 \pm 294.7$ \\
\hline $\begin{array}{c}\text { Duration of labor }{ }^{1^{*}}(\min ) \\
\text { Mean } \pm S D\end{array}$ & & $637.4 \pm 417.1$ & $423.3 \pm 386.7$ \\
\hline $\begin{array}{l}\text { Amount of the intrapartum } \\
\text { Hemorrhage(g), Mean } \pm \text { SD }\end{array}$ & & $551.6 \pm 285.2$ & $625.9 \pm 311.3$ \\
\hline \multirow{2}{*}{ Delivery style } & Vaginal & 5 & 7 \\
\hline & Cesarean section & 2 & 2 \\
\hline \multirow{10}{*}{$\begin{array}{l}\text { Type of disorder } \\
\text { (There is overlapping) }\end{array}$} & Depression & 2 & 5 \\
\hline & Panic disorder & 2 & 2 \\
\hline & Eating disorder & 1 & 1 \\
\hline & Anxiety disorder & 1 & 1 \\
\hline & PTSD & 0 & 1 \\
\hline & Bipolar disorder & 0 & 1 \\
\hline & Adjustment disorder & 1 & 0 \\
\hline & $\begin{array}{l}\text { Emotionally unstable } \\
\text { personality disorder }\end{array}$ & 0 & 1 \\
\hline & $\begin{array}{c}\text { After effects of } \\
\text { stimulant poisoning }\end{array}$ & 1 & 0 \\
\hline & Schizophrenia & 1 & 0 \\
\hline
\end{tabular}

${ }^{\star} 1$ only vaginal delivery.

subjects was $29.0 \pm 7.0$ years old in primiparas and $32.0 \pm 4.0$ years old in multiparas. The most common mental disorder was depression.

Five of the participants received medication during pregnancy (1 primipara, 4 multiparas), and 7 received medication during postpartum hospitalization (4 primiparas, 3 multiparas). Among those who did not take medication during pregnancy and restarted or started medication after childbirth and during postpartum hospitalization were all primiparas ( 3 women).

\subsection{Characteristics of Feeling of Postpartum Fatigue}

Figure 1 shows the mean total and subscale scores of change in the PFS points in primiparas and multiparas. The primiparas' PFS score was the highest on day 1 , 


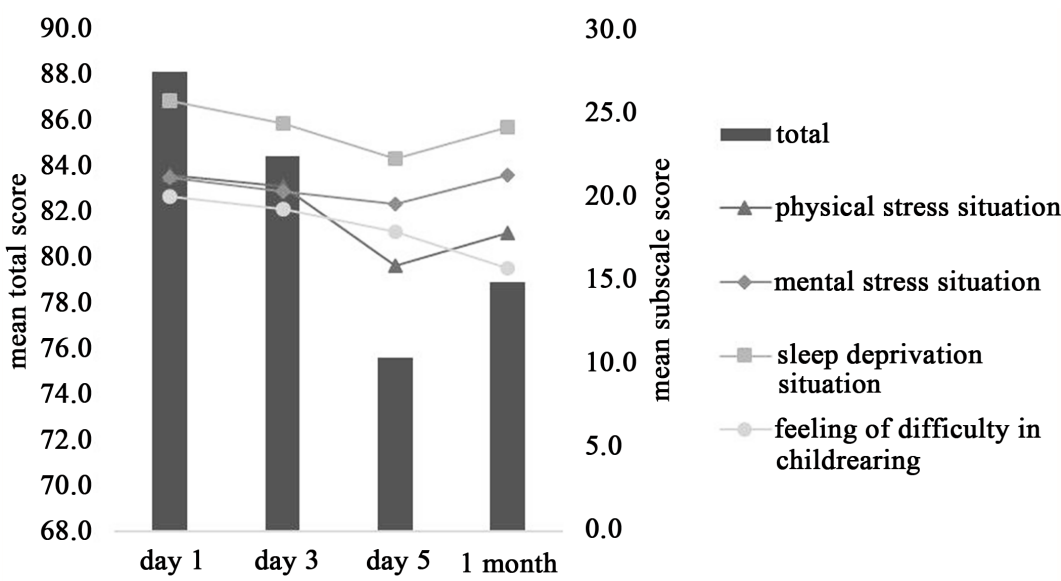

(a)

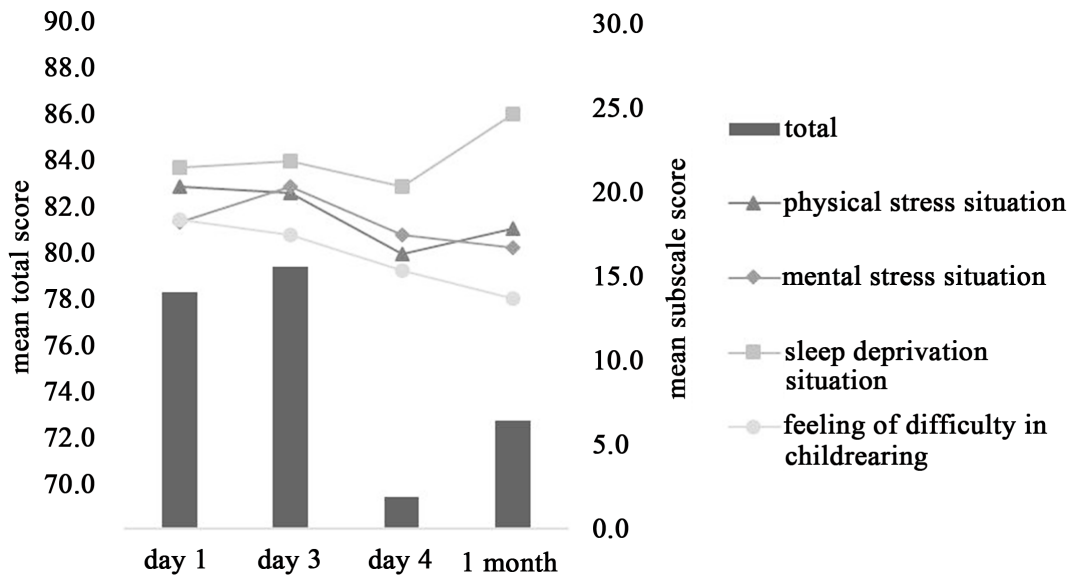

(b)

Figure 1. The change in mean scores of the PFS total and subscales. (a): Primiparas; (b): Multiparas.

and the mean score was $88.1 \pm 15.7$ points. In multiparas, the PFS score became the highest on day 3 , and the mean score was $79.4 \pm 16.4$ points.

\subsection{Characteristics of Depression}

Figure 2 shows the mean scores of EPDS and percentage of women, both primiparas and multiparas, who scored 9 or more points.

\subsection{Relationship between Fatigue and Depression}

In primiparas, the EPDS score at 1 month has a positive correlation with the PFS total point $(\mathrm{r}=0.880 / p=0.021)$ on day 3 and the "mental stress situation" $(\mathrm{r}=$ $0.818 / p=0.047)$ at 1 month. In multiparas, the EPDS score at 1 month has a positive correlation with the PFS total point on day $4(\mathrm{r}=0.734 / p=0.024)$ and 1 month $(\mathrm{r}=0.863 / p=0.003)$, "physical stress situation" on day $4(\mathrm{r}=0.843 / p=$ $0.004)$ and 1 month $(\mathrm{r}=0.829 / p=0.006)$, "mental stress situation" at 1 month ( $\mathrm{r}$ $=0.965 / p=0.000)$, and "sleep deprivation situation" at 1 month $(\mathrm{r}=0.674 / p=$ $0.046)$. 


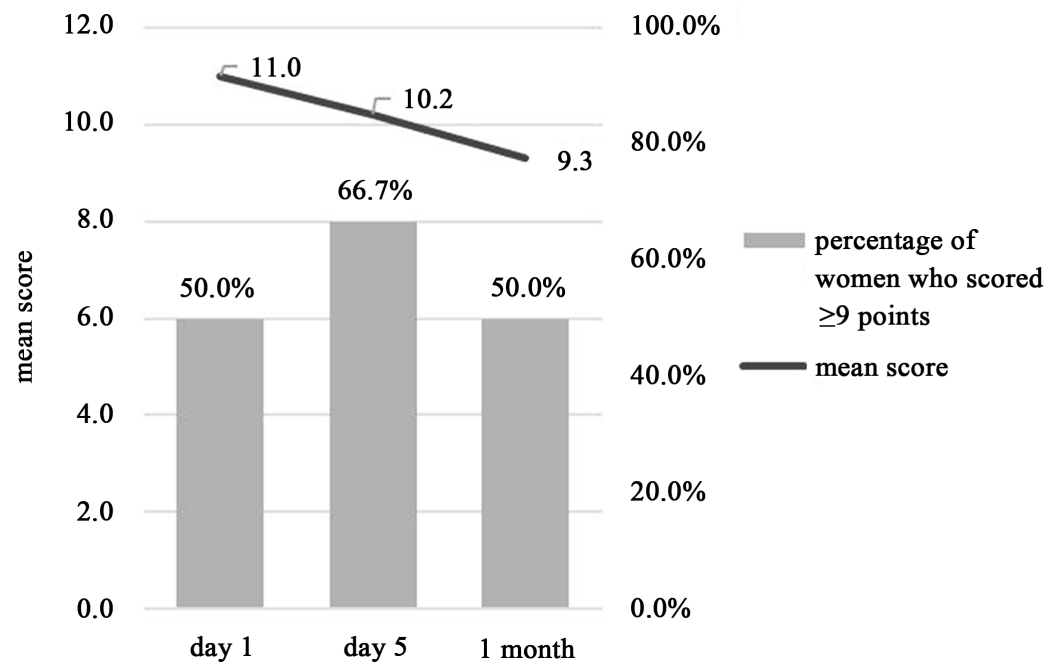

(a)

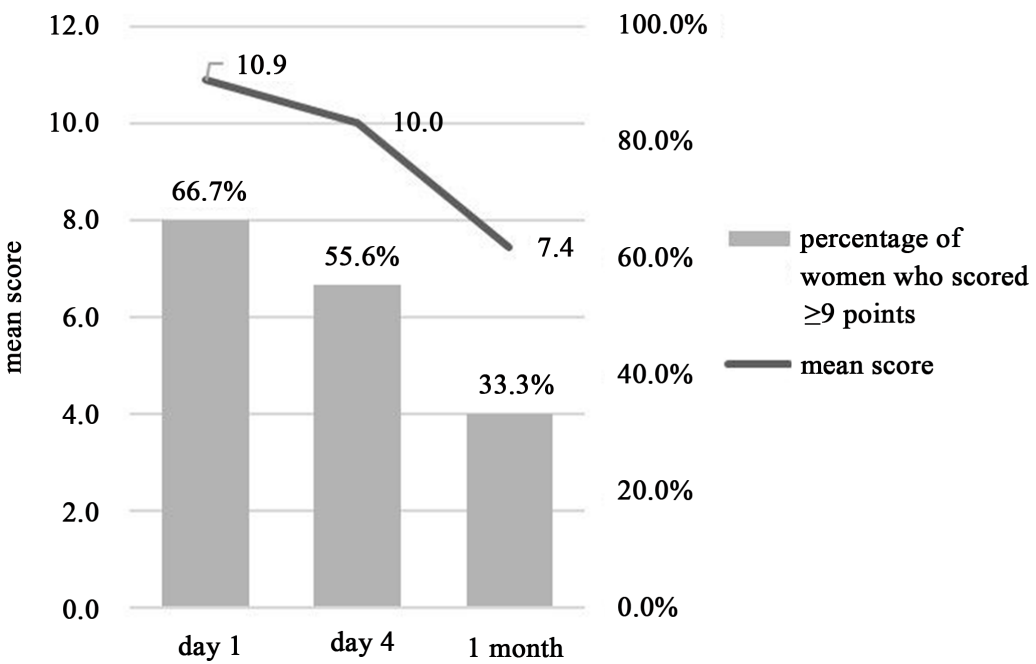

(b)

Figure 2. Change in mean scores of EPDS and percentage of women who scored $\geq 9$ points. (a): Primiparas; (b): Multiparas.

\section{Discussion}

In a study conducted by Yamazaki et al. [10], the average score of the PFS of women with mental disorders was $82.00 \pm 19.64$ points. The subjects of this study had somewhat higher scores especially on the first day. However, when compared with the multiparas' scores, the primiparas' scores were high throughout the investigation period. In Yamazaki et al.'s report, these were the scores on the second day after rooming-in, so it seemed like a feeling of fatigue at that time when the mothers were mastering child-rearing skills. It was noted that it is a primipara woman as one of the enhancing factors of fatigue [10]. We found that this could also apply to women with mental disorders, especially on the first day after birth, as mothers are still unfamiliar with handling newborns and physical fatigue due to delivery remains. Therefore, it was presumed that the 
score of primiparas became higher than what was given in Yamazaki et al.'s report. The total PFS score of both primiparas and multiparas, gradually descending during postpartum hospitalization, had risen in 1 month. In a survey targeting older primiparas using the same scale, it was found that the total score of the PFS gradually decreased from hospitalization with rooming-in to 1 month [11]. Although the scale used for measuring the feeling of fatigue is different, the previous study consistently investigated 0 to 28 days after birth and found that the feeling of fatigue decreases gradually in women who did not have a depression tendency [7]. Therefore, it can be said that one of the characteristics of women with mental disorders was that the feeling of fatigue, which had been decreasing until discharge, increased in 1 month after birth.

In a previous study [12] targeting Japanese mothers who had no history of mental disorders, the proportion of women whose EPDS scores were 9 or more on both 5 days and 1 month after birth was about 16\%. However, in our study, the percentage of postpartum women who had higher EPDS scores on day 1 had these high scores for 1 month. Since there is a risk of postpartum depression in all psychiatric disorders, it seems inevitable that the proportion at which the EPDS becomes high is more. However, depending on the delivery history, there was a difference in the proportion of high scorers and the relevance to fatigue.

In primiparas, the proportion of high EPDS scorers reaches its maximum on day 5 , half of the primiparas exceeded the cutoff value even at 1 month, and the mean score exceeds 9 points. The relationship between the EPDS scores at 1 month and the PFS "mental stress situation" was positively correlated. There are cases in which primiparas have started or restarted medication for mental disorders during postpartum hospitalization and it is estimated that symptoms of mental disorders such as severe anxiety and depression after birth are more likely to be worse than in the case of multiparas. In contrast, the primiparas will experience breast-feeding for the first time, along with the disorder, and in some cases continue with oral treatment. Even though primiparas are unfamiliar with newborns, it is possible that these burdens overlap in a synergistic manner causing mental stress and influencing the EPDS scores.

In the case of multiparas, the EPDS score was going down, and the percentage of high scorers also decreased as the number of days after birth increased. However, since this was related to the feeling of fatigue even at 1 month, it could be said that depression is severe in the case of a multipara with fatigue. It was seen that the EPDS scores for 1 month and the total of the PFS and subscales' scores, other than "feeling of difficulty in childrearing", were related; especially, "sleep deprivation situation" score at 1 month was higher than those during hospitalization. While in hospital, there is the assistance of a midwife, and so it is easy to get sleep and rest, but in the case of a multipara, after discharge they also have to take care of their other children, leaving little time for rest.

\section{Clinical Implications}

From the above, in the case of primiparas, there is a possibility that the symp- 
toms of the disorder itself deteriorate. After discharge, we will need to give advice according to the family background and symptoms of the disorder in each woman, and consult a psychiatric department from the time of hospitalization, if required. In multiparas, to ensure necessary sleep and rest after discharge, it is suggested that it is important to understand the family support system until discharge, or a person who supports in child rearing. For women who are unlikely to receive any assistance, it will become necessary to examine their mental condition regularly.

\section{Limitations}

One limitation of this study is that the number of subjects is small and there is not enough data to generalize. For a more reliable survey it will be necessary to focus on postpartum women with specific mental disorders and include more subjects.

The second is that it includes women who have undergone a cesarean section (CS) operation. In the report by Tsuchiya et al. [6] that compared for the presence or absence of depression tendency, no significant relationship was seen during postpartum hospitalization after a vaginal delivery, selective CS, and emergency CS, but in 1 month after birth, the proportion of depressed tendency was high among women who had undergone emergency CS. It is unlikely that CS itself has a big influence on EPDS, as in the case of selective CS fatigue is not related to EPDS even during postpartum hospitalization for a month. However, since the body invasion and recovery processes differ from vaginal delivery, there is a possibility that fatigue in CS cases may have been affected to a considerable extent. When increasing the number of cases, it is necessary to consider the kind of delivery and mental condition of the woman and its relationship with fatigue and depression.

\section{References}

[1] The Ministry of Health, Labor and Welfare (2016) The 6th Review Conference on Perinatal Medical System. http:/www.mhlw.go.jp/stf/shingi2/0000134653.html

[2] Sasaki, A., Iwasa, K., Matsuo, S., Hirasugi, Y., Iwasaku, K. and Kitawaki, J. (2012) Perinatal Management of Pregnant Women with Mental Illness. Japanese Society of Psychosomatic Obstetrics and Gynecology, 17, 206-212.

[3] Mori, E. (2014) Developing Nursing Guidelines for Childrearing Support in Japanese Older Primiparas. http://www.n.chiba-u.jp/mamatasu/doc/guidelines_fix.pdf

[4] Japan Society of Obstetrics and Gynecology/Japan Association of Obstetricians and Gynecologists (2017) Guideline for Obstetrical Practice in Japan. Japan Society of Obstetrics and Gynecology, Tokyo.

[5] The Ministry of Health, Labor and Welfare (2014) Outline of Patient Survey in 2014, Statistics Table. http://www.mhlw.go.jp/toukei/saikin/hw/kanja/14/dl/toukei.pdf

[6] Tsuchiya, M., Mori, E., Iwata, H., Aoki, K., Maehara, K., Sakajo, A. and Ozawa, H. (2016) Factors Associated with Depressive Symptomology in Mothers with a Psychiatric History during the 1 month Postpartum. Japanese Journal of Maternal 
Health, 57, 90-15.

[7] Bozoky, I. and Corwin, E.J. (2002) Fatigue as a Predictor of Postpartum Depression. Journal of Obstetric Gynecologic \& Neonatal Nursing, 31, 436-443. https://doi.org/10.1111/j.1552-6909.2002.tb00066.x

[8] Yamazaki, K., Takagi, H. and Saito, M. (2015) Development of a Scale for a "Feeling of Postpartum Fatigue" Evoked in Early Puerperium and Verification of the Reliability and Validity. Japanese Journal of Maternal Health, 55, 711-720.

[9] Okano, T., Murata, M., Masuji, A., Tamaki, R., Nomura, J., Miyaoka H. and Kitamura, T. (1996) Reliability and Validity of the Edinburgh Postpartum Depression Scale (EPDS) in Japan version. Archives of Psychiatric Diagnostics and Clinical Evaluation, 7, 525-533.

[10] Yamazaki, K., Takagi, H., Kubo, K., and Masuda, T. (2016) A Study on Fatigue during the Early Puerperium and its Associated Aggravating Factor. Japanese Jour nal of Maternal Health, 57, 314-322.

[11] Yamazaki, K. (2017) Relationship between a Feeling Fatigue and Depressive State in Late-Birth Primiparas during One Month after Childbirth. Journal of the Japan Maternal and Infant Care Association, 10, 51-59.

[12] Ishikawa, N., Goto, S., Murase, S., Kanai, A., Masuda T., Aleksic, B., Usui, H. and Ozaki, N. (2011) Prospective Study of Maternal Depressive Symptomatology among Japanese Women. Journal of Psychosomatic Research, 71, 264-269.

https://doi.org/10.1016/j.jpsychores.2011.02.001 